www.nature.com/pj

\title{
Synthesis, characterization and applications of amphiphilic elastomeric polyurethane networks in drug delivery
}

\author{
Pablo Christian Caracciolo ${ }^{1}$, Cristina Sanz Pita ${ }^{2}$, Gustavo Abel Abraham ${ }^{1}$, José Alberto Méndez ${ }^{2}$ and \\ Jordi Gironès Molera ${ }^{3}$
}

Polyurethanes have a key role in the development of many different biomedical devices because of their exceptional biocompatibility, mechanical properties and versatility. Although linear segmented polyurethanes have been extensively studied, the investigation of cross-linked polyurethanes remains limited. In this work, three series of polyurethane networks were synthesized by reacting poly(ethylene glycol) and hexamethylene diisocyanate with either tetrafunctional poloxamer Tetronic 701 or poly( $\varepsilon$-caprolactone)s triol with molecular weights of 1060 and $3130 \mathrm{Da}$. The hydrophilic/hydrophobic ratio was varied by selecting the appropriate amounts of monomers, and its effects on the swelling behavior and the thermal properties were analyzed. Studies concerning the release of a model drug were performed, the results of which indicate that these materials hold promise for use in controlled implantable drug-delivery devices and antimicrobial coatings.

Polymer Journal (2013) 45, 331-338; doi:10.1038/pj.2012.131; published online 4 July 2012

Keywords: controlled drug release; hydrophilic/hydrophobic ratio; polyurethane networks; swelling behavior; thermal properties

\section{INTRODUCTION}

Since the 1960s, polyurethanes (PURs) have played a key role in the development of biomedical devices because of their versatility, mechanical properties and excellent blood compatibility. PURs have been extensively studied as biodegradable and/or injectable materials and have been used in several applications. ${ }^{1-4}$ Linear PURs are commonly obtained by the reaction of a macrodiol, a diisocyanate and a short diol or diamine chain extender, with an equimolar ratio of hydroxyl to isocyanate groups. Otherwise, polyurethane networks (PUNs) are synthesized by incorporating at least one reactant with functionality higher than two. Depending on their composition, PURs can be hydrophilic/hydrophobic, elastomeric/rigid and degradable/non-degradable. ${ }^{1,5}$

Although linear segmented PURs and multiblock copolymers with controlled hydrophilicity have been extensively studied, ${ }^{5-8}$ the investigation of amphiphilic PUNs is still limited. ${ }^{9}$ PUNs composed of hydrophilic and hydrophobic segments can provide controlled swelling and enable the homogeneous dispersion of drugs. ${ }^{10}$ For this reason, the characteristics of PUNs are ideal for use in controlled drug-release systems. However, their use in this field remains largely unexplored. ${ }^{11-13}$
Thermoresponsive hydrogels have great potential to be used in regenerative medicine because they can solidify in situ via a fast and mild process to form three dimensional scaffolds when the temperature is adjusted to physiological conditions. ${ }^{14}$ At certain critical temperatures, commonly referred to as lower or upper critical solution temperature, changes in the overall hydrophilicity of some polymers cause a decrease in their solubility. As a result of the conformational changes occurring at this temperature, a reversible physical crosslinking of the polymer occurs (commonly referred to as sol-gel transition or gelation). Over the past years, these copolymers have been extensively studied for applications that include drug and gene delivery, burn wound covering and bone tissue repair, as evidenced by the numerous works on these particular systems. ${ }^{14-17}$

In recent years, in an effort to design biodegradable thermosensitive systems, attention has been centered on the development of amphiphilic block copolymers based on poly(ethylene glycol) (PEG) ${ }^{18-20}$ focusing particularly on the ones containing biodegradable polymers, such as poly(lactic acid), poly(glycolic acid) or poly(E-caprolactone) (PCL). ${ }^{21-24}$

PEG exhibits outstanding physicochemical properties, a lack of toxicity and good biocompatibility, and minimizes non-specific protein adsorption and cell adhesion. Because of these properties,

\footnotetext{
${ }^{1}$ Instituto de Investigaciones en Ciencia y Tecnología de Materiales, INTEMA (UNMdP-CONICET), Mar del Plata, Argentina; ${ }^{2}$ Lepamap Group, Universitat de Girona, Campus Montilivi, Girona, Spain and ${ }^{3}$ Nanomateriales Poliméricos y Biomateriales, Instituto de Ciencia y Tecnología de Polímeros (ICTP-CSIC), Madrid, Spain

Correspondence: Dr PC Caracciolo, Instituto de Investigaciones en Ciencia y Tecnología de Materiales, INTEMA (UNMdP-CONICET), Avenue Juan B, Justo 4302, B7608FDQ Mar del Plata, Argentina.

Email: pcaracciolo@fi.mdp.edu.ar

or Dr JG Molera, Instituto de Ciencia y Tecnología de Polímeros (ICTP), Consejo Superior de Investigaciones Científicas (CSIC), C/Juan de la Cierva, 3, 28006 Madrid, Spain. Email: jgirones@ictp.csic.es
}

Received 5 March 2012; revised 25 April 2012; accepted 3 May 2012; published online 4 July 2012 
PEG is considered to be a very suitable material for the engineering of bioactive scaffolds and was selected as the hydrophilic macrodiol for PUNs prepared in this study. Meanwhile, PCL is a linear biocompatible and bioresorbable polyester whose utilization as a biomedical material has been extensively investigated. PCL exhibits a hydrophobic character due to the presence of five methylene groups and a single relatively polar ester group in each repeating unit. Multi-arm PCL macromolecules can also be prepared using initiators with functionalities higher than two. In this work, two star-shaped PCLs with low molecular weights were synthesized and employed as hydrophobic segments and crosslinking agents. Thus, two series of PUNs containing PCL and PEG were prepared.

In addition, to introduce thermoresponsive features, the utilization of poloxamines (or tetronics), presenting four poly(ethylene oxide)poly(propylene oxide) (PEO-PPO) blocks joined together by a central ethylene diamine bridge, was studied. By adjusting the hydrophobic (PPO) to hydrophilic (PEO) block ratio, molecular weight and concentration, the gelation of these thermoresponsive copolymers can be tuned to occur at physiological temperature and $\mathrm{pH} .^{25-27}$ Thus, a hydrophobic tetrafunctional block copolymer, named Tetronic 701, and PEG were employed to obtain a thermoresponsive series of PUNs.

In this work, the effect of composition on the swelling behavior and the morphology of the PUNs and their possible application as drugdelivery vehicles is investigated.

\section{MATERIALS AND METHODS}

\section{Materials}

Glycerol, $\varepsilon$-caprolactone ( $\varepsilon$-CL), stannous 2-ethyl hexanoate (stannous octoate, $\left.\mathrm{Sn}(\mathrm{Oct})_{2}\right)$, Tetronic 701 (number-average molecular weight $(\mathrm{Mn})=3600 \mathrm{Da}$ ), PEG $(\mathrm{Mn} \approx 400 \mathrm{Da})$, 1,6-hexamethylene diisocyanate (HDI), acetaminophen (paracetamol), and $N, N$-dimethylacetamide (DMAc) were purchased from Sigma-Aldrich (St Louis, MO, USA). DBTDL (Dibutyltin dilaurate) was purchased from Fluka (Buchs, Switzerland). The alcohols were dried under vacuum at $60^{\circ} \mathrm{C}$ for $24 \mathrm{~h}$ before use. DMAc was dried over molecular sieves $(4 \AA)$ and distilled at $50^{\circ} \mathrm{C}$ under reduced pressure. The other reagents were used as received.

\section{Polyurethane networks}

Synthesis of PCL triol. Initiated by glycerol, two PCL triols were obtained by ring-opening polymerization of $\varepsilon$-caprolactone in bulk. The monomer and the initiator were placed in a three-necked round-bottomed flask together with $0.1 \% \mathrm{w} / \mathrm{w}$ of stannous octoate acting as a catalyst. The amount of initiator was regulated to control the final average molecular weight of the polymers ([ $\varepsilon$ CL:glycerol]: [6:1] and [24:1] for PCL triols named $\mathrm{PCL}_{780}$ and $\mathrm{PCL}_{2830}$, respectively). Polymerizations were performed under $\mathrm{N}_{2}$ atmosphere at $130{ }^{\circ} \mathrm{C}$ for $24 \mathrm{~h}$ and constant magnetic stirring (Figure 1). The products were then dissolved in chloroform, precipitated in cold hexane and recovered by filtration. Finally, PCL triol was dried under vacuum at room temperature until a constant weight was achieved.

Synthesis of PUNs. Predetermined amounts of polyol (either Tetronic 701 or PCL triol) and chain extender (PEG) were placed in a test tube and dissolved in DMAc. After adding $0.1 \% \mathrm{w} / \mathrm{w}$ of DBTDL, acting as catalyst, together with $\mathrm{HDI}$, the reactive mixture was vigorously stirred and degassed under vacuum for $5 \mathrm{~min}$. Films were then prepared by casting from the degassed colorless mixtures onto Teflon molds and allowed to cure at $60^{\circ} \mathrm{C}$ for $24 \mathrm{~h}$. Finally, the resulting PUNs were dried at room temperature under vacuum to remove the residual solvent.

All of the synthesized PUNs comply with the following molar ratio:

$$
\left([\mathrm{OH}]_{\mathrm{polyol}}+[\mathrm{OH}]_{\mathrm{PEG}}\right) \cdot 1.05=[\mathrm{NCO}]_{\mathrm{HDI}}
$$

The hydroxyl component contained 10, 30, 50, 70, 90 and 100\% by weight of polyol (either Tetronic 701 or PCL triol). Samples were coded as TX-Y, $P_{780} X-Y$ or $P_{2830} X-Y$, where $T, P_{780}$ and $P_{2830}$ correspond to Tetronic 701 , and PCL triol with [ $[\varepsilon-C L$ :glycerol]: [6:1] and [24:1], respectively, while $X$ and $Y$ correspond to the mass percentages of polyol and PEG, respectively.

Synthesis of drug-loaded polyurethane networks (DPUNs). The physical loading did not always lead to a homogeneous distribution of drugs. The presence of a hydroxyl functional group in the chemical structure of paracetamol and the hydrolytic instability of the polyester allowed the drug was to be chemically coupled to the PUN through a biodegradable linkage. Predetermined amounts of paracetamol together with polyol (either Tetronic 701 or PCL triol) and chain extender (PEG) were placed in a test tube and dissolved in DMAc, according to the procedure previously described.

All synthesized DPUNs comply with the following molar ratio

$$
\left([\mathrm{OH}]_{\text {paracetamol }}+[\mathrm{OH}]_{\text {polyol }}+[\mathrm{OH}]_{\mathrm{PEG}}\right) \cdot 1.05=[\mathrm{NCO}]_{\mathrm{HDI}}
$$

Samples were coded as TX- $Y-A, P_{780} X-Y$ - $A$ or $P_{2830} X-Y-A$, where T, $P_{780}$ and $P_{2830}$ represent the polyol used in the DPUN preparation, $X$ corresponds to Tetronic or PCL triol weight percentage, $Y$ represents the PEG weight percentage and $A$ indicates the loaded mass of paracetamol. For example, a sample named T90-10-25 corresponds to a DPUN based on $90 \% \mathrm{w} / \mathrm{w}$ Tetronic, $10 \% \mathrm{w} / \mathrm{w}$ PEG and $25 \mathrm{mg}$ of paracetamol per gram of DPUN.

\section{Characterization of monomers and networks}

Physicochemical characterization. The number-average molecular weight (Mn) values of PCL triol employed in the synthesis of the PUNs were determined by an end-group titration procedure described in ASTM D4274-99. ${ }^{28}$ Three titration values were averaged. ${ }^{1} \mathrm{H}$ nuclear magnetic resonance (NMR) spectroscopy was performed using a Bruker AM-300 NMR spectrometer (Bruker Co., Medford, MA, USA). All spectra were obtained at room temperature from $5 \% \mathrm{w} / \mathrm{v} \mathrm{CDCl}_{3}$ solutions and used a delay time between pulses of $5 \mathrm{~s}$. Infrared analysis was conducted at room temperature using a Nicolet 6700 FTIR spectrometer (Nicolet Instrument Inc.,
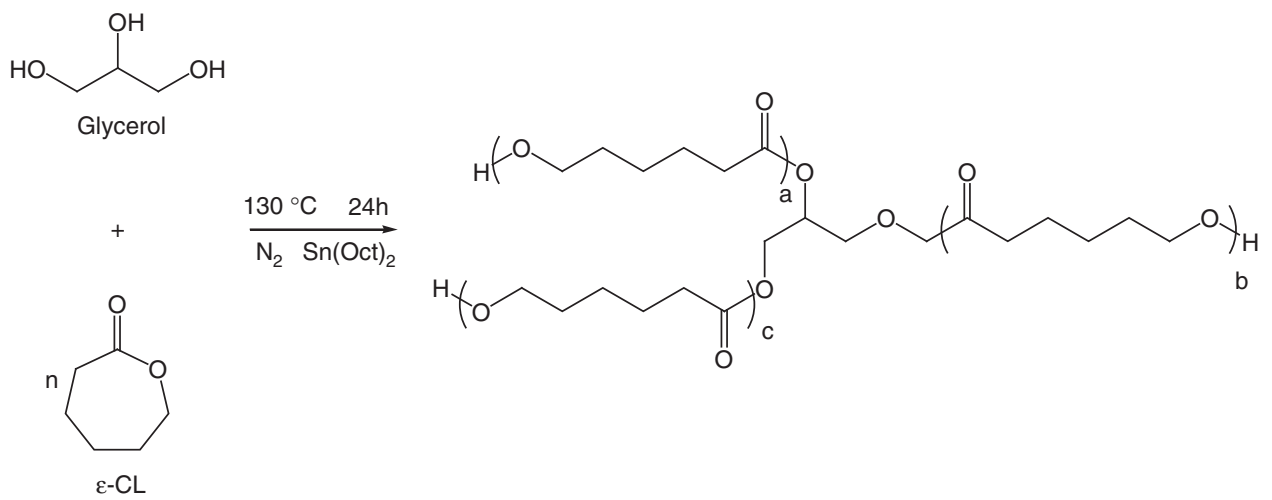

Figure 1 Polymerization of e-CL initiated by glycerol. 
Madison, WI, USA) equipped with an ATR system. FTIR spectra were collected from 64 scans at $2 \mathrm{~cm}^{-1}$ resolution. Thermal characterization was performed using a Shimadzu DSC-50 (Shimadzu Co., Tokyo, Japan) under nitrogen atmosphere. Experiments were conducted from -120 to $250^{\circ} \mathrm{C}$ at a heating rate of $10^{\circ} \mathrm{C} \mathrm{min}^{-1}$. The glass transition temperature $\left(T_{\mathrm{g}}\right)$ was measured at the onset of the transition. The degree of crystallinity $\left(X_{\mathrm{c}}\right)$ was calculated taking into account the melting enthalpy for $100 \%$ crystalline PCL $\left(148.05 \mathrm{Jg}^{-1}\right){ }^{29}$

The morphology of the PUN was studied by scanning electron microscopy (SEM JEOL Model JSM-6460LV Denton Vacuum Desk II, JEOL USA, Inc., Peabody, MA, USA). The specimens were cut with a razorblade, and the new surfaces were coated with $\mathrm{Au} / \mathrm{Pd}$ before being imaged by scanning electron microscope.

To test the soluble fraction and the dimensional stability of the PUNs, disk specimens of $1.25 \mathrm{~mm}$ in thickness and $10 \mathrm{~mm}$ in diameter were cut from the PUN films.

The soluble fraction of the PUN was determined by immersing the previously weighed disk samples in distilled water at $37^{\circ} \mathrm{C}$ for $24 \mathrm{~h}$ with regular water changes. Afterwards, the samples were freeze-dried in a Benchtop 2.0 K liofilizer (Virtis SP Industries, Gardiner, NY, USA). The soluble fraction was calculated from the following equation:

$$
\text { Sol fraction }(\%)=\frac{m_{0}-m_{\mathrm{f}}}{m_{0}} \cdot 100
$$

where $m_{0}$ is the initial weight of the sample and $m_{\mathrm{f}}$ is the weight of the sample after extraction of the soluble fraction. At least three sample values were averaged.

The dimensional stability of PUN at different temperatures $(5,15,25$ and $37^{\circ} \mathrm{C}$ ) was determined by placing the disks in a phosphate buffered solution (PBS) for certain periods of time. Then, the swollen samples were wiped gently with filter paper, to remove the surface water, and weighed. The mass change resulting from the water uptake was calculated according to the following equation:

$$
\text { Swelling }(\%)=\frac{m_{\mathrm{t}}-m_{0}}{m_{0}} \cdot 100
$$

where $m_{\mathrm{t}}$ is the weight of the sample after a certain time of swelling and $m_{0}$ is the weight of the dry sample $(t=0)$ after extraction of the soluble fraction. At least three samples were analyzed for each time and temperature.

\section{Determination of drug content and drug-release profiles}

Drug-release measurements were performed on extracted samples (network fraction) in $\mathrm{PBS}$ at $37^{\circ} \mathrm{C}$. At regular intervals, aliquots were extracted and replaced with fresh PBS, and the drug content was determined by UV-vis (Agilent 8453, Santa Barbara, CA, USA) spectrometer measurements at $243 \mathrm{~nm}$. Assays were performed to ensure sink conditions. The results are the average of three samples. Analysis of variance statistical analysis $(P=0.05)$ was conducted to determine the significance of the differences observed between the populations of the results.

\section{RESULTS AND DISCUSSION}

\section{Tetronic 701-based PUN}

Characterization of Tetronic 701. ${ }^{1} \mathrm{H}$ NMR results provided structural information on the polyol. There was no evidence of free amino groups, indicating that only four-arm species are present. The analysis revealed that propylene glycol is the most abundant monomer in the
Tetronic structure, with an approximate ratio of PPO:PEO (4:1) (Table 1). Otherwise, the arms were found not to exhibit a homogeneous structure; thus, there are both PPO and PEO chainended arms (see the supplementary material for further details). The thermal analysis showed the amorphous morphology of the polyol.

PUNs containing Tetronic 701 (TX-Y series). PUNs with different Tetronic to PEG ratios were successfully synthesized. The absence of unreacted isocyanate groups in the obtained PUN films was confirmed by infrared analysis.

Table 2 shows the soluble fraction for the PUN series prepared from Tetronic 701. Given the low solubility of Tetronic 701 at room temperature and its thermal-responsive character, the soluble fractions were analyzed at different temperatures. As shown in Table 2, the soluble fraction increased with extraction temperature and PEG content. In fact, when this hydrophilic component represented more than $50 \% \mathrm{w} / \mathrm{w}$, the PUN could not sustain its integrity during the extraction process at extraction temperatures higher than $15^{\circ} \mathrm{C}$.

In addition, note that in contrast to Tetronic, the solubility of PEG in water increases with temperature. Thus, the soluble fraction could be attributed to the presence of -(PEG-HDI $)_{n^{-}}$oligomers.

Dimensional stability. Figure 2 shows the water absorption curves for the T80-20 network at different temperatures. The thermosensitive character of Tetronic 701 is showed by the higher water absorption values observed at lower temperatures. In fact, although swelling equilibria were not achieved until $4-5 \mathrm{~h}$ at temperatures below the lower critical solution temperature of Tetronic $\left(16-18^{\circ} \mathrm{C}\right)$, PUN experienced a very fast swelling at $5{ }^{\circ} \mathrm{C}$, leading to a water uptake value close to $200 \%$ in just $1 \mathrm{~h}$. The evolution of water uptake at $15^{\circ} \mathrm{C}$ was similar to that at $5^{\circ} \mathrm{C}$, although the kinetics involved were much slower. In clear contrast, when the experiments were conducted at

Table 2 Soluble fraction (\%) of Tetronic-based PUN at different temperatures

\begin{tabular}{lcccr}
\hline & $\begin{array}{c}\text { Soluble } \\
\text { fraction (\%) } \\
\text { at } 37^{\circ} \mathrm{C}\end{array}$ & $\begin{array}{c}\text { Soluble } \\
\text { fraction (\%) } \\
\text { at } 25^{\circ} \mathrm{C}\end{array}$ & $\begin{array}{c}\text { Soluble } \\
\text { fraction (\%) } \\
\text { at } 15^{\circ} \mathrm{C}\end{array}$ & $\begin{array}{c}\text { Soluble } \\
\text { fraction (\%) } \\
\text { at } 5{ }^{\circ} \mathrm{C}\end{array}$ \\
\hline T100-0 & $3.97 \pm 0.01$ & $3.56 \pm 0.02$ & $3.34 \pm 0.06$ & $3.65 \pm 0.04$ \\
T90-10 & $9.90 \pm 0.01$ & $7.08 \pm 0.02$ & $5.15 \pm 0.05$ & $4.83 \pm 0.04$ \\
T80-20 & $11.69 \pm 0.02$ & $8.54 \pm 0.03$ & $8.76 \pm 0.02$ & $9.05 \pm 0.03$ \\
T70-30 & $11.77 \pm 0.09$ & $10.87 \pm 0.07$ & $4.00 \pm 0.01$ & $4.07 \pm 0.02$ \\
T60-40 & $13.80 \pm 0.05$ & $13.31 \pm 0.09$ & $10.23 \pm 0.04$ & $8.92 \pm 0.05$ \\
T50-50 & $14.05 \pm 0.08$ & $13.82 \pm 0.06$ & $12.16 \pm 0.01$ & $11.22 \pm 0.03$ \\
T40-60 & - & - & $14.13 \pm 0.06$ & $13.99 \pm 0.02$ \\
T30-70 & - & - & - & $24.66 \pm 0.09$ \\
T20-80 & ND & ND & ND & ND \\
T10-90 & ND & ND & ND & ND \\
\hline
\end{tabular}

Abbreviations: ND, not determined; PUN, polyurethane networks.

\begin{tabular}{|c|c|c|c|c|c|c|c|c|c|}
\hline \multirow[b]{2}{*}{ Polyol } & \multirow[b]{2}{*}{$M n_{\text {com }}(D a)$} & \multicolumn{2}{|c|}{ Relative monomer abundance (\%) } & \multicolumn{2}{|c|}{ Terminal groups (\%) } & \multirow[b]{2}{*}{$M n_{N M R}(D a)$} & \multicolumn{3}{|c|}{$D S C$} \\
\hline & & PEO & PPO & PEO & PPO & & $T_{g}\left({ }^{\circ} \mathrm{C}\right)$ & $T_{m}\left({ }^{\circ} \mathrm{C}\right)$ & $X_{c}(\%)$ \\
\hline Tetronic 701 & 3600 & 19.6 & 80.4 & 61.3 & 38.7 & 2860 & -69.7 & - & - \\
\hline
\end{tabular}

Table 1 Characterization of Tetronic 701 


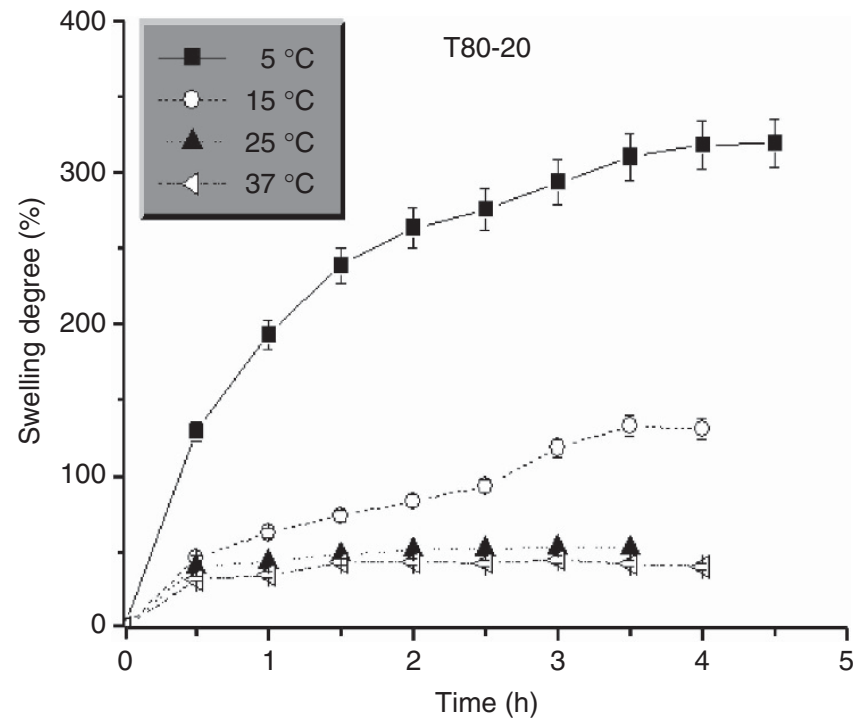

Figure 2 Effect of temperature on the water absorption curves of T80-20.

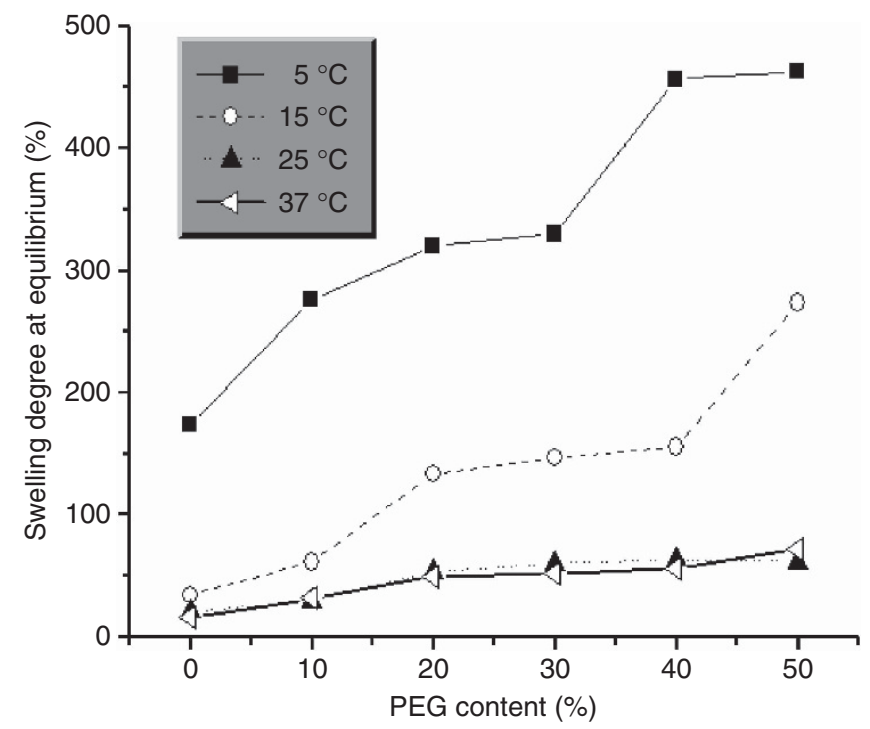

Figure 3 Equilibrium swelling degree for TX-Y networks at different temperatures.

temperatures above the lower critical solution temperature of Tetronic, the swollen degrees exhibited significantly lower values, and the PUNs reached their swelling equilibria within the first hour. In addition, no significant differences were observed between the equilibrium swelling degree of the PUNs at 25 and $37^{\circ} \mathrm{C}$.

The effect of the content of the hydrophilic component (PEG) on the equilibrium swelling of PUN is represented in Figure 3. The high crosslinking density and high hydrophobic character of pure Tetronic networks is reflected in the low swelling degree of T100-0. Owing to its hydrophilic character, increasing the PEG content caused a continuous and progressive increase in the swelling degree of PUN, especially at low temperatures. Thus, whilst T100-0 swelled more than $170 \%$ at $5{ }^{\circ} \mathrm{C}$, T50-50 increased its weight by more than $460 \%$. In comparison, at $37^{\circ} \mathrm{C}$ the same two formulations swelled $\sim 15$ and $70 \%$, respectively.

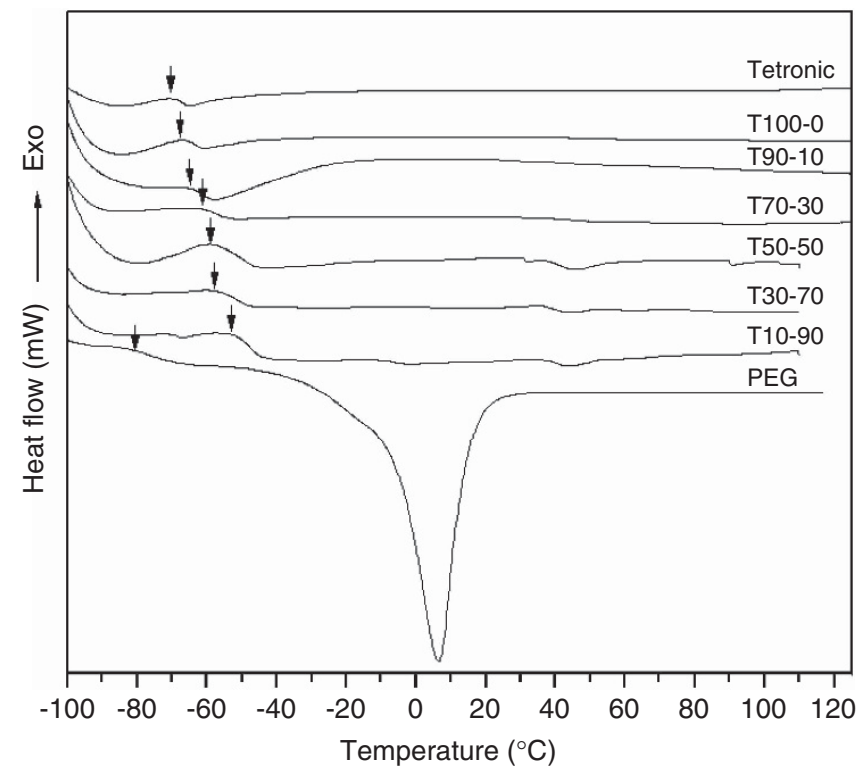

Figure 4 DSC thermograms of TX-Y PUN and their components.

Thermal properties. Figure 4 shows the DSC thermograms for the TX-Y PUN series and its major components (Tetronic 701 and PEG chain extender). Only a single-glass transition temperature $\left(T_{\mathrm{g}}\right)$ could be observed for each PUN. The absence of a macrodiol led to polymeric network structures without hard and soft microdomains in this series.

Pure Tetronic and PEG exhibited $T_{\mathrm{g}}$ values at -69.7 and $-83.9^{\circ} \mathrm{C}$, respectively. Owing to crosslinking, the $T_{\mathrm{g}}$ values of all the obtained PUNs were higher than those of pure Tetronic and of PEG. Depending on the Tetronic to PEG ratio, the $T_{\mathrm{g}}$ of the PUN ranged from -67 to $-50{ }^{\circ} \mathrm{C}$, with changes in heat capacities increasing with PEG content. Despite expectations, $T_{\mathrm{g}}$ values increased with increasing PEG content in the series. This behavior could be associated to the increase in the chain length of the -(PEG-HDI $)_{n}$ - segments connecting the Tetronic monomers. PEG and HDI are connected through urethane groups in the $-(\mathrm{PEG}-\mathrm{HDI})_{\mathrm{n}^{-}}$segments, so the increase in chain length leads to an increase in hydrogen-bonding interaction. Finally, although there was an increase in chain length between points of chemical crosslinking, the appearance of a higher fraction of reversible physical crosslinking led to an increase in $T_{\mathrm{g}}$ with PEG content.

PUNs with PEG content less than 50\% exhibited no endothermal peak, indicating the amorphous nature of these PUN formulations. Otherwise, DPUNs did not display any signals corresponding to paracetamol and exhibited similar thermal responses (data not shown).

Drug-release profiles of DPUNs containing Tetronic 701 (TX-Y-A series). Either in the form of a foam, injectable scaffold, or film, PURs have been widely examined for several biomedical applications, such as bone and cartilage tissue engineering, ${ }^{30}$ vascularization $^{31}$ and nerve regeneration. ${ }^{32}$ In this work, we evaluated an easy way to incorporate drugs into the structure of PURs to limit the inflammatory responses of the body.

PUNs with PEG content less than 30\% exhibited low soluble fractions that could easily be removed from the networks due to their high swelling behavior at low temperatures. In addition, this process 
also ensures the full removal of any toxic component (traces of unreacted reagents, solvent and catalyst) while preserving the main structural integrity of the networks.

DPUNs with formulations T90-10 and T80-20 were polymerized in the presence of $25 \mathrm{mg}$ of paracetamol per gram of DPUN. Once prepared, the release of the drug was analyzed under physiological conditions. Figure 5 shows the release profile over time of paracetamol from the obtained DPUNs during the stripping with PBS. The data are reported as the concentration of drug per gram of tested DPUN. Drug-release profiles showed a fast release of paracetamol during the first 3-4h, after which they evolved in a much slower progressive manner. In both cases, drug release was observed until the

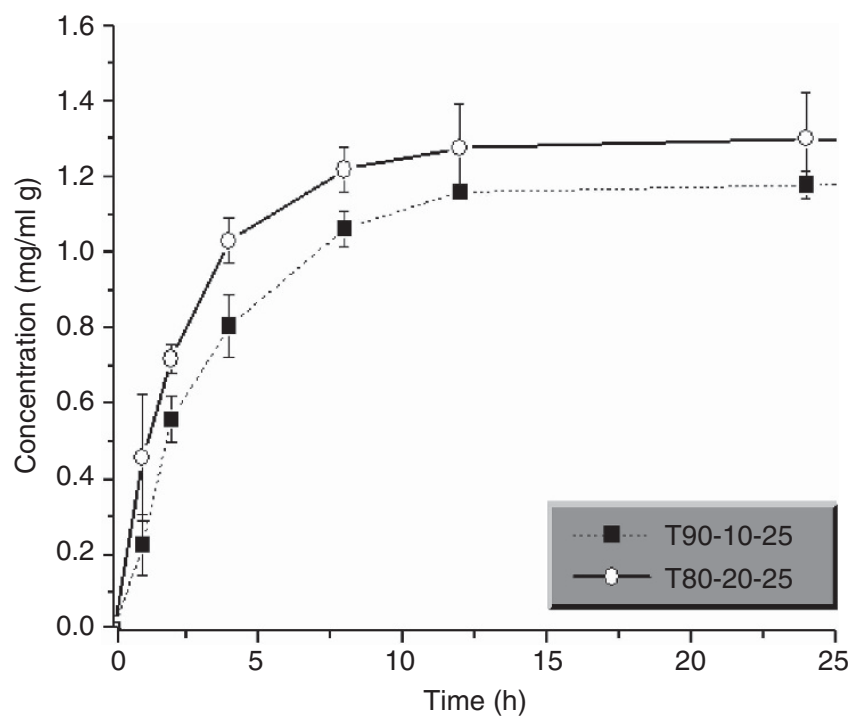

Figure 5 Drug-release profile of loaded TX-Y. first $24 \mathrm{~h}$. After this period, the concentration of the drug remained constant and no further drug was released by any of the analyzed systems. The DPUNs with higher PEG content exhibited slightly faster kinetics, although no significant differences in the total amount of released drug could be detected (Table 3). After 6 days of immersion, the total amount of paracetamol released by the analyzed DPUNs was $\sim 4 \mathrm{mg}$ per gram of PUN, far from the $25 \mathrm{mg}$ that was loaded. Although only a fraction of the drug was released during the assayed time, the remaining drug is expected to be released along with further hydrolytic degradation of the bulk of the DPUN.

\section{PCL triol-based PUNs}

Characterization of PCL triol. Two PCL triols with hydroxyl chain end groups were synthesized by ring-opening polymerization of $\varepsilon-\mathrm{CL}$ initiated by glycerol in the presence of stannous octoate as a catalyst (Figure 1). Glycerol acts as an initiator of the polymerization, controlling the number of arms of the star-shaped polymers and also the molecular weight by the adjustment of the [ $\varepsilon$-CL:glycerol] ratio. PCL triols with [ $\varepsilon$-CL:glycerol] ratios of [6:1] and [24:1] $\left(\mathrm{PCL}_{780}\right.$ and $\mathrm{PCL}_{2830}$, respectively) were synthesized with yields $>90 \%$. ${ }^{1} \mathrm{H}$ NMR measurements provided structural information on the PCL triols. Previous studies ${ }^{33}$ showed that at [ $\varepsilon$-CL:glycerol] feed ratios lower than [30:1] the reaction product is composed by one-, two- and three-arm species, increasing the relative abundance of the most-substituted glycerol species with the increase in [ $\varepsilon$-CL:glycerol] feed ratio. Following the analysis previously described, the ${ }^{1} \mathrm{H}$ NMR spectra of the PCL triols revealed the presence of three different species of substituted glycerol units for both PCL triols (Table 4). As expected, $\mathrm{PCL}_{2830}$ exhibited a higher content in the trisubstituted species than $\mathrm{PCL}_{780}$, but a slower content in mono- and disubstituted species.

To obtain stoichiometric PUNs, the monomer concentrations must be adjusted. For this reason, the molecular weights of the synthesized PCL triols were determined as accurately as possible before starting the synthesis of the PUN. ${ }^{34}$ The Mn determined by end-group

Table 3 Paracetamol release of PUN tested in PBS at $37^{\circ} \mathrm{C}$

\begin{tabular}{|c|c|c|c|c|c|}
\hline PUN poliol & PUN code & $\begin{array}{l}\text { Normalized drug } \\
\text { loading }(\mathrm{mg})\end{array}$ & $\begin{array}{l}\text { Normalized dose } e^{\mathrm{b}} \\
\left(m g h^{-1}\right)\end{array}$ & $\begin{array}{c}\text { Normalized equilibrium drug } \\
\text { concentration }{ }^{a}\left(\mathrm{mg} \mathrm{m}^{-1}\right)\end{array}$ & $\begin{array}{c}\text { Normalized total drug } \\
\text { released }(\mathrm{mg})\end{array}$ \\
\hline \multirow[t]{2}{*}{ Tetronic } & T90-10-25 & 25 & 0.60 & $1.23 \pm 0.04$ & $3.8 \pm 0.1$ \\
\hline & T80-20-25 & 25 & 0.77 & $1.29 \pm 0.07$ & $3.9 \pm 0.2$ \\
\hline \multirow[t]{4}{*}{$\mathrm{PCL}_{2830}$ triol } & P90-10-10 & 10 & 0.43 & $0.64 \pm 0.03$ & $2.0 \pm 0.1$ \\
\hline & P80-20-10 & 10 & 0.65 & $0.85 \pm 0.02$ & $2.7 \pm 0.1$ \\
\hline & P90-10-25 & 25 & 0.81 & $1.24 \pm 0.15$ & $3.7 \pm 0.3$ \\
\hline & P80-20-25 & 25 & 0.77 & $1.41 \pm 0.02$ & $4.2 \pm 0.1$ \\
\hline
\end{tabular}

Abbreviations: PBS, phosphate buffered solution; PCL, poly( $\varepsilon$-caprolactone); PUN, polyurethane networks.

aDrug values per gram of PUN.

${ }^{b}$ Drug-dose release was calculated from the lineal region of the drug-release profiles, typically the initial $4 \mathrm{~h}$.

Table 4 Characterization of synthesized PCL triol, $\mathrm{PCL}_{780}$ and $\mathrm{PCL}_{2830}$

\begin{tabular}{|c|c|c|c|c|c|c|c|c|c|}
\hline \multirow[b]{2}{*}{ PCL triol } & \multirow[b]{2}{*}{$M n_{t}(D a)$} & \multirow[b]{2}{*}{$M n_{\text {tit }}(D a)$} & \multicolumn{3}{|c|}{ Number of arms (\%) } & \multirow[b]{2}{*}{$M n_{N M R}(D a)$} & \multicolumn{3}{|c|}{$D S C$} \\
\hline & & & 1 & 2 & 3 & & $T_{g}\left({ }^{\circ} \mathrm{C}\right)$ & $T_{m}\left({ }^{\circ} \mathrm{C}\right)$ & $X_{c}(\%)$ \\
\hline $\mathrm{PCL}_{780}$ & 777 & 1060 & 20.1 & 46.4 & 33.5 & 1170 & -74.5 & 47.8 & 47.7 \\
\hline $\mathrm{PCL}_{2830}$ & 2832 & 3130 & 1.1 & 30.6 & 68.3 & 4130 & -64.3 & 54.3 & 68.1 \\
\hline
\end{tabular}

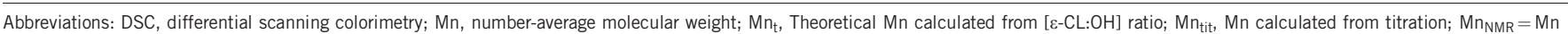
calculated from ${ }^{1} \mathrm{H}$ NMR spectra; NMR, nuclear magnetic resonance; PCL, poly( $\varepsilon$-caprolactone); $T_{\mathrm{g}}$, glass transition temperature; $X_{\mathrm{c}}$, degree of crystallinity. 
Table 5 Soluble fraction (\%) of PCL-based PUN at $37^{\circ} \mathrm{C}$

\begin{tabular}{lcc}
\hline & \multicolumn{2}{c}{ Soluble fraction (\%) } \\
\cline { 2 - 3 } Sample & $P U N_{780}$ & PUN 2830 \\
\hline P100-0 & 4.7 & 1.1 \\
P90-10 & 4.6 & 1.6 \\
P70-30 & 13.3 & 10.7 \\
P50-50 & 18.2 & 17.0 \\
P30-70 & 20.5 & 16.0 \\
P10-90 & ND & ND \\
\hline
\end{tabular}

Abbreviations: ND, not determined; PCL, poly(ع-caprolactone); PUN, polyurethane networks.

Table 6 Equilibrium swelling degree of PCL triol-based PUN in PBS at $37^{\circ} \mathrm{C}$

\begin{tabular}{lcr}
\hline & \multicolumn{2}{c}{ Equilibrium swelling degree (\%) } \\
\cline { 2 - 3 } Sample & $P C L_{780}$ & $P C L_{2830}$ \\
\hline P100-0 & 4.5 & 3.3 \\
P90-10 & 9.1 & 9.6 \\
P70-30 & 27.2 & 28.4 \\
P50-50 & 43.8 & 45.2 \\
P30-70 & 64.3 & 65.5 \\
\hline
\end{tabular}

Abbreviations: PBS, phosphate buffered solution; PCL, poly( $\varepsilon$-caprolactone); PUN, polyurethane networks.

titration are more accurate than that estimated by NMR measurements. For this reason, the first ones were employed in PUN formulations. The obtained values for $\mathrm{PCL}_{780}$ and $\mathrm{PCL}_{2830}$ were 1060 and $3130 \mathrm{Da}$, respectively. Both PCLs were semicrystalline, and the thermal properties are summarized in Table 4.

PUNs containing PCL triols ( $P X-Y$ series). In addition to the Tetronic 701-based networks, a biodegradable PUN series prepared from PCL triols were also studied. PEG was used as a chain extender to obtain amphiphilic block copolymers. Two series of PUNs, varying the PCL to PEG ratio and employing PCL triols with different $\mathrm{Mn}\left(\mathrm{PUN}_{780}\right.$ and $\mathrm{PUN}_{2830}$ ), were successfully synthesized. The complete reaction of the isocyanate groups was confirmed by the absence of the isocyanate peak at $2250-2270 \mathrm{~cm}^{-1}$ in the infrared spectrum of the obtained PUN films. As for the TX-Y networks, the soluble fraction increased with increasing proportion of PEG in both series (Table 5). In addition, networks with PEG content $>70 \%$ underwent partial dissolution during extraction. The use of $\mathrm{PCL}_{780}$ versus $\mathrm{PCL}_{2830}$ in the PUN formulation seemed to increase the soluble fraction at the same PCL to PEG ratio.

Dimensional stability. Studies showed that water uptake was exclusively controlled by the content of hydrophilic monomer (PEG) and was independent of the chain length and the degree of crystallinity of the hydrophobic monomer (Table 6). Thus, for the same PEG content, both PX-Y series exhibited similar values of equilibrium swelling. In addition, no differences were observed when conducting tests at different temperatures (data not shown). In all cases, equilibrium was achieved in $\sim 1 \mathrm{~h}$. Moreover, after $3 \mathrm{~h}$ of immersion, the first signs of hydrolytic degradation became noticeable in the formulations with higher PEG content.
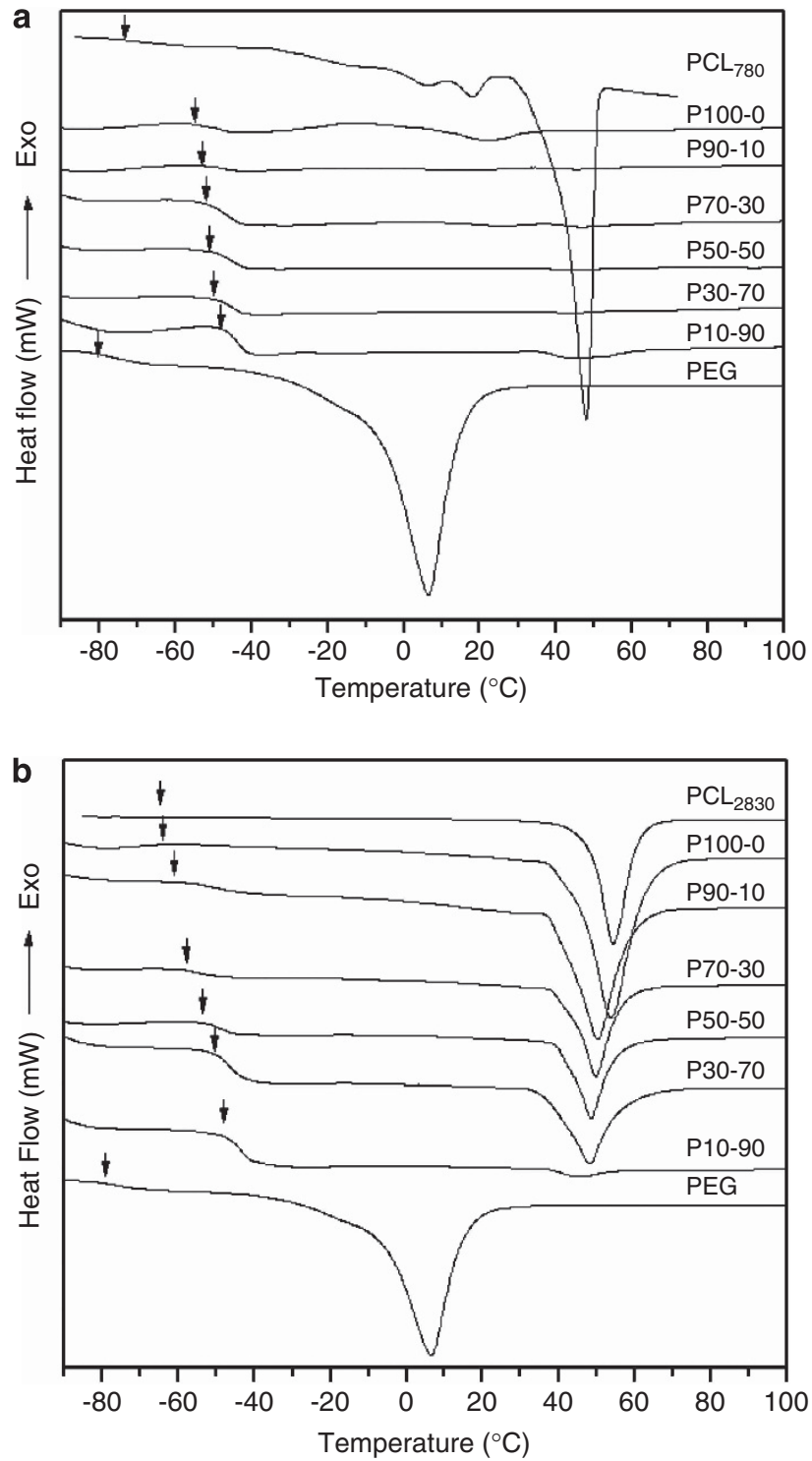

Figure 6 DSC thermograms of PUN based on PCL2830 (a), PCL780 (b) and their individual constituents.

Thermal properties. Figure 6 shows the non-normalized DSC thermograms of $\mathrm{PUN}_{780}$ and $\mathrm{PUN}_{2830}$, both PCL triols and PEG. The DSC analysis revealed the presence of a single $T_{\mathrm{g}}$ in both series. As mentioned for the TX-Y PUN series, the absence of a macrodiol in the PUN formulation led to networks without microphase segregation.

Pure $\mathrm{PCL}_{780}, \mathrm{PCL}_{2830}$ and PEG have $T_{\mathrm{g}}$ values at $-74.5,-64.3$ and $-83.9^{\circ} \mathrm{C}$, respectively. Due to chemical crosslinking, both series of PUN exhibited higher $T_{\mathrm{g}}$ values than the ones of the corresponding PCL triol and PEG. Although the chain length between crosslinks increased with increasing PEG content, $T_{\mathrm{g}}$ also displayed this behavior. As with Tetronic-based PUNs, the higher content of urethane groups led to higher hydrogen-bonding interaction. Thus, in spite of a lower chemical crosslinking density, higher reversible physical crosslinking led to higher $T_{\mathrm{g}}$ values. This phenomenon was more evident for the $\mathrm{PUN}_{2830}$ series, while the $\mathrm{PUN}_{780}$ series exhibited a slight increase in $T_{\mathrm{g}}$ values with an increase in the PEG 


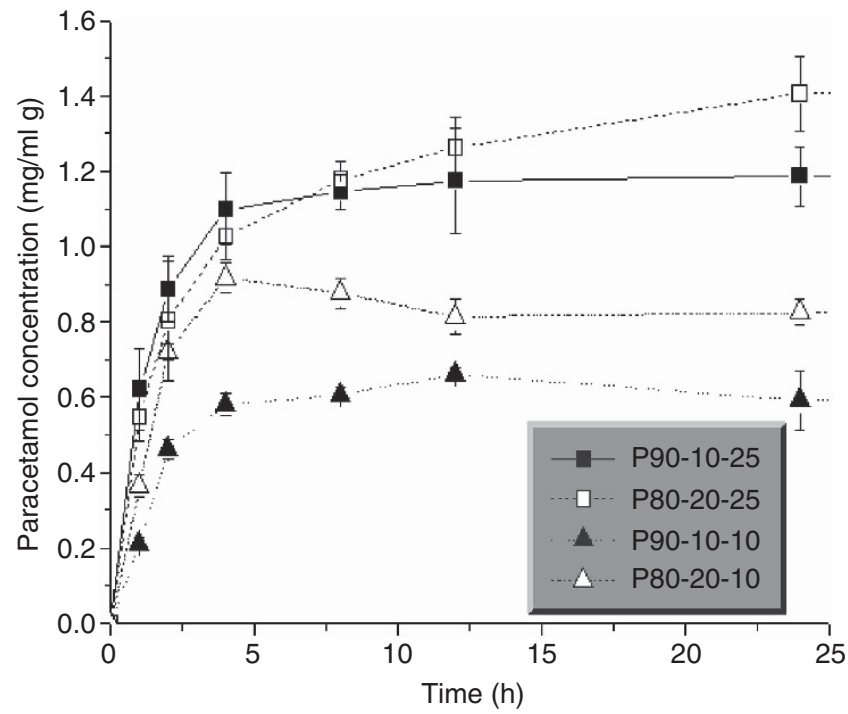

Figure 7 Drug-release profiles of PX-Y loaded with 10 and $25 \mathrm{mg}$ of paracetamol.

composition. In addition, $\mathrm{PUN}_{2830}$ had lower $T_{\mathrm{g}}$ values than those observed for the PUN $\mathrm{P}_{780}$ series for the same PEG content. Additionally, the same $T_{\mathrm{g}}$ value was found for both P10-90 samples, suggesting that, at low PCL content, the thermal behavior becomes independent of the PCL chain length because physical crosslinking is the dominant factor.

In the $\mathrm{PUN}_{780}$ series, only $\mathrm{P}_{780} 100-0$ formulations exhibited a small melting endotherm, whereas the rest of the formulations were amorphous. These results indicate that network formation, aided by phase mixing, resulted in impeded crystallization of semyicrystalline PCL. Otherwise, the higher crystallinity of $\mathrm{PCL}_{2830}$ relative to $\mathrm{PCL}_{780}$ led to the $\mathrm{PUN}_{2830}$ series exhibiting noticeable melting endotherms directly dependent on PCL content.

For the TX-Y PUN series, the DPUNs did not exhibit any signals corresponding to paracetamol and had similar thermal responses (data not shown).

Drug-release profiles of PCL triol-based DPUNs (PX-Y-A series). As both PX-Y series exhibited similar swelling behavior for the same PEG content, only formulations of $\mathrm{PCL}_{2830}$ triol-based PUN series were selected for performing drug-release studies. Similar to the Tetronicbased systems, $P_{2830} 90-10$ and $P_{2830} 80-20$ networks were tested as drug carriers. Figure 7 shows the drug-release profiles of the $P_{2830} 90-10$ and $P_{2830} 80-20$ networks, both polymerized with 10 and $25 \mathrm{mg}$ of paracetamol per gram of DPUN. The paracetamol release is observed to be slightly faster in the compositions that include higher amounts of PEG in their formulation. In addition, the total amount of drug released is also higher in the more hydrophilic networks.

For the $P_{2830} 90-10-10$ and $P_{2830} 80-20-10$ systems, the concentrations of paracetamol in the stripping solutions attain their maximum after $12 \mathrm{~h}$ before stabilizing at 0.64 and $0.85 \mathrm{mg} \mathrm{ml}^{-1}$ per gram of DPUN sample, respectively. The higher equilibrium swelling degree of PUNs containing larger amounts of hydrophilic PEG, which allows a faster and higher water penetration, relates well with the observed profiles of drug release.

Although formulations loaded with $25 \mathrm{mg}$ of paracetamol per gram of DPUN followed similar kinetics (PX-Y-25 series), the stabilization of drug concentrations in the stripping solutions were not attained until $24 \mathrm{~h}$. Thus, after $24 \mathrm{~h}$, the drug concentrations were 1.24 and

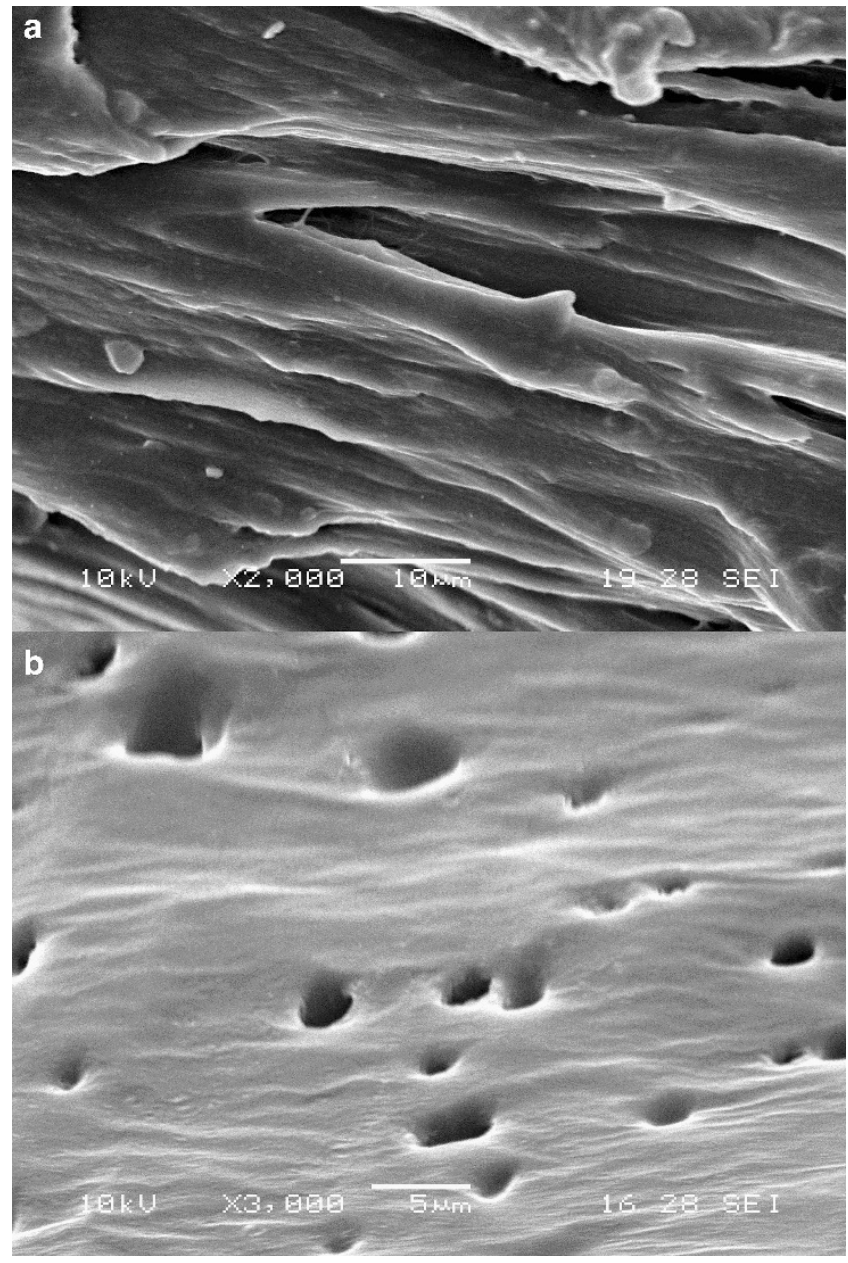

Figure 8 SEM microphotographs of PUN loaded with paracetamol: (a) T9010-25 (b) P90-10-25.

$1.41 \mathrm{mg} \mathrm{ml}^{-1}$ for $P_{2830} 90-10-25$ and $P_{2830} 80-20-25$, respectively. As for the $P_{2830} X-Y-10$ formulations, the total amount of paracetamol released was slightly higher for the more hydrophilic $P_{2830} 80-20-25$ formulation. Although this behavior could be assigned to the higher water uptake of formulations with larger PEG ratio, no significant differences could be detected in terms of the release kinetics (Table 3).

A comparison of the scanning electron microscopy micrographs of the Tetronic and the PCL-based networks (Figure 8) indicates that PCL-based DPUNs exhibit higher surface porosity. This phenomenon can be attributed to the higher viscosity of PCL compared with that of Tetronic, which limits pore occlusion during casting. In turn, the viscosity of PCL triols required the utilization of larger amounts of solvent, whose evaporation also contributed to the formation of higher surface porosity. The higher surface porosity enables more rapid water penetration, which explains the more rapid drug-release kinetics of the PX- $Y$ networks and their higher total drug release.

The observed release profiles could only be attributed to superficial hydrolytic degradation despite the presence of the hydrophilic soft segments. Thus, after 1 month of testing, the total released drug did not reach $25 \%$ of the total loaded amount.

\section{CONCLUSIONS}

Three series of PUNs prepared from thermoresponsive Tetronic 701 or PCL triol, PEG and HDI were successfully synthesized and 
characterized. The PUNs with PEG content less than 30\% exhibited low soluble fractions that were easily removable from the networks because of their high swelling degree. In the case of Tetronic-based PUNs, the sol fraction could be removed at low temperature. This feature ensured the full removal of the toxic components whilst preserving its main structural integrity. Drugs could be chemically incorporated into the PUNs during the synthesis stage. Biodegradable PUNs prepared from PCL triol chains extended with PEG exhibited the typical thermal response of copolymers. As in the case of TX-Y-A networks, $P_{2830} X-Y$-A exhibited a homogeneous drug distribution and a controlled drug-release profile that was dependent upon the drug concentration during loading and the PEG content. On the basis of these results, the PUNs studied in this work should generate considerable interest as candidate materials for non-toxic drugdelivery implantable devices.

1 Guelcher, S. A. Biodegradable polyurethanes: Synthesis and applications in regenerative medicine. Tissue Eng. Part B 14, 3-17 (2008).

2 Puppi, D., Chiellini, F., Piras, A. M. \& Chiellini, E. Polymeric materials for bone and cartilage repair. Progr. Polym. Sci. 35, 403-440 (2010).

3 Gorna, K. \& Gogolewski, S. Biodegradable porous polyurethane scaffolds for tissue repair and regeneration. J. Biomed. Mater. Res. 79A, 128-138 (2006).

4 Caracciolo, P. C., Thomas, V., Vohra, Y. K., Buffa, F. \& Abraham, G. A. Electrospinning of novel biodegradable poly(ester urethane)s and poly(ester urethane urea)s for soft tissue-engineering applications. J. Mater. Sci. Mater. Med. 20, 2129-2137 (2009).

5 Bonzani, I. C., Adhikari, R., Houshyar, S., Mayadunne, R., Gunatillake, P. \& Stevens, M. M. Synthesis of two-component injectable polyurethanes for bone tissue engineering. Biomaterials 28, 423-433 (2007).

6 Abraham, G. A., Marcos-Fernandez, A. \& San Roman, J. Bioresorbable poly(ester-ether urethane)s from L-lysine diisocyanate and triblock copolymers with different hydrophilic character. J. Biomed. Mater. Res. 76A, 729-736 (2006).

7 Cohn, D., Lando, G., Sosnik, A., Garty, S. \& Levi, A. PEO-PPO-PEO-based poly(ether ester urethane)s as degradable reverse thermo-responsive multiblock copolymers. Biomaterials 27, 1718-1727 (2006).

8 Fromstein, J. D. \& Woodhouse, K. A. Elastomeric biodegradable polyurethane blends for soft tissue applications. J. Biomater. Sci. Pol. Ed. 13, 391-406 (2002).

9 Phung, H., Schacht, E., Du Prez, F., Gelan, J., Adriaesens, P. \& Storme, L. Crosslinked polyurethane-based gels. Pol. J. 35, 353-358 (2003).

10 Bruin, P., Smedinga, J., Pennings, A. J. \& Jonkman, M. F. Biodegradable lysine diisocyanate-based poly(glycolide-co- $\varepsilon$-caprolactone)-urethane network in artificia skin. Biomaterials 11, 291-295 (1990).

11 Hafeman, A. E., Zienkiewicz, K. J., Carney, E., Litzner, B., Stratton, C., Wenke, J. C. \& Guelcher, S. A. Local Delivery of Tobramycin from Injectable Biodegradable Polyurethane Scaffolds. J. Biomater. Sci. Pol. Ed. 21, 95-112, 2010).

12 Sivak, W. N., Zhang, J., Petoud, S. \& Beckman, E. J. Simultaneous drug release at different rates from biodegradable polyurethane foams. Acta Biomater. 5, 2398-2408 (2009).

13 Sivak, W. N., Pollack, I.F., Petoud, S., Zamboni, W. C., Zhang, J. \& Beckman, E. J. Catalyst-dependent drug loading of LDI-glycerol polyurethane foams leads to differing controlled release profiles. Acta Biomater. 4, 1263-1274 (2008).

14 Klouda, L. \& Mikos, A. G. Thermoresponsive hydrogels in biomedical applications. Eur. J. Pharm. Biopharm. 68, 34-45 (2008).
15 Kabanov, A. V., Lemieux, P., Vinogradov, S. \& Alakhov, V. Pluronic(R) block copolymers: novel functional molecules for gene therapy. Adv. Drug Deliv. Rev. 54, 223-233 (2002).

16 Yoshida, T., Aoyagi, T., Kokufuta, E. \& Okano, T. Newly designed hydrogel with both sensitive thermoresponse and biodegradability. J. Polym. Sci. A Polym. Chem. 41, 779-787 (2003).

17 Qiu, Y. \& Park, K. Environment-sensitive hydrogels for drug delivery. Adv. Drug Deliv. Rev. 53, 321-339 (2001).

$18 \mathrm{He}, \mathrm{G}$., Ma, L. L., Pan, J. \& Venkatraman, S. ABA and BAB type triblock copolymers of PEG and PLA: A comparative study of drug release properties and 'stealth' particle characteristics. Int. J. Pharm. 334, 48-55 (2007).

19 Lochmann, A., Nitzsche, H., von Einem, S., Schwarz, E. \& M $\Sigma$ der, K. The influence of covalently linked and free polyethylene glycol on the structural and release properties of rhBMP-2 loaded microspheres. J. Control. Release 147, 92-100 (2010).

20 Vermonden, T., Fedorovich, N. E., van Geemen, D., Alblas, J., van Nostrum, C. F., Dhert, W. J. A. \& Hennink, W. E. Photopolymerized thermosensitive hydrogels: Synthesis, degradation, and cytocompatibility. Biomacromolecules 9, 919-926 (2008).

21 Dumas, J. E., Davis, T., Holt, G. E., Yoshii, T., Perrien, D. S., Nyman, J. S., Boyce, T. \& Guelcher, S. A. Synthesis, characterization, and remodeling of weight-bearing allograft bone/polyurethane composites in the rabbit. Acta Biomater. 6, 2394-2406 (2010)

22 Gorna, K. \& Gogolewski, S. In vitro degradation of novel medical biodegradable aliphatic polyurethanes based on epsilon-caprolactone and Pluronics $(R)$ with various hydrophilicities. Pol. Degr. Stab. 75, 113-122 (2002).

23 Cohn, D. \& Hotovely-Salomon, A. Biodegradable multiblock PEO/PLA thermoplastic elastomers: molecular design and properties. Polymer 46, 2068-2075 (2005).

24 Clapper, J. D., Skeie, J. M., Mullins, R. F. \& Guymon, C. A. Development and characterization of photopolymerizable biodegradable materials from PEG-PLA-PEG block macromonomers. Polymer 48, 6554-6564 (2007).

25 Moghimi, S. M. \& Hunter, A. C. Poloxamers and poloxamines in nanoparticle engineering and experimental medicine. Trends Biotechnol. 18, 412-420 (2000).

26 Bonacucina, G., Spina, M., Misici-Falzi, M., Cespi, M., Pucciarelli, S., Angeletti, M. \& Palmieri, G. F. Effect of hydroxypropyl [beta]-cyclodextrin on the self-assembling and thermogelation properties of Poloxamer 407. Eur. J. Pharm. Sci. 32, 115-122 (2007).

27 Rodrigues, J. d. \& Nascimento, R. S. Synthesis and rheological behavior of hydrophobically modified block copolymers. J. Appl. Polym. Sci. 116, 3047-3055 (2010).

28 ASTM D 4274-99: Standard Test Methods for Testing PU Raw Materials: Determination of Hydroxyl Numbers of Polyols (2012).

29 van Krevelen, D. W. \& te Nijenhuis, K. Properties of Polymers: Their Correlation with Chemical Structure; their Numerical Estimation and Prediction from Additive Group Contributions, 4th edn (Elsevier, Amsterdam, The Netherlands, 2009).

30 Grad, S., Kupcsik, L., Gorna, K., Gogolewski, S. \& Alini, M. The use of biodegradable polyurethane scaffolds for cartilage tissue engineering: potential and limitations. Biomaterials 24, 5163-5171 (2003).

31 Laschke, M. W., Strohe, A., Scheuer, C., Eglin, D., Verrier, S., Alini, M., Pohlemann, T. \& Menger, M. D. In vivo biocompatibility and vascularization of biodegradable porous polyurethane scaffolds for tissue engineering. Acta Biomater. 5, 1991-2001 (2009).

32 Borkenhagen, M. Stoll, R. C., Neuenschwander, P., Suter, U. W. \& Aebischer, P. In vivo performance of a new biodegradable polyester urethane system used as a nerve guidance channel. Biomaterials 19, 2155-2165 (1998).

33 Lang, M., Wong, R. P. \& Chu, C. C. Synthesis and structural analysis of functionalized poly ( $\varepsilon$-caprolactone)-based three-arm star polymers. J. Polym. Sci. A Polym. Chem. 40, 1127-1141 (2002)

34 Marcos-Fernandez, A., Abraham, G. A., Valentin, J. L. \& San Roman, J. Synthesis and characterization of biodegradable non-toxic poly(ester-urethane-urea)s based on poly( $\varepsilon$ caprolactone) and amino acid derivatives. Polymer 47, 785-798 (2006).

Supplementary Materials accompanies the paper on Polymer Journal website (http://www.nature.com/pj) 